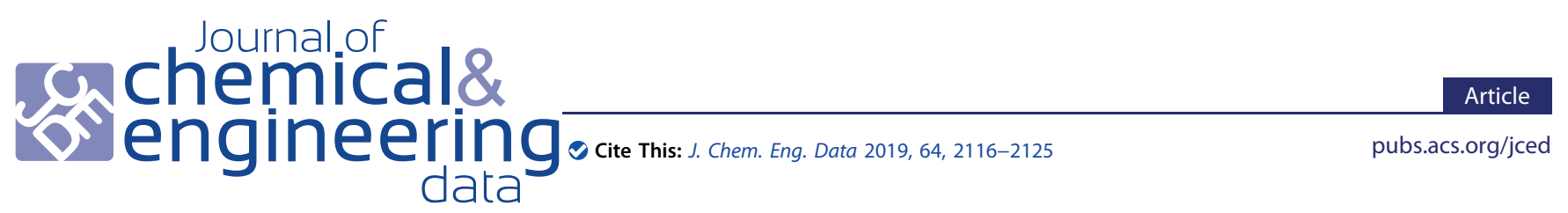

\title{
Phase Behavior for the System Carbon Dioxide + p-Nitrobenzaldehyde: Experimental and Modeling
}

Tecio Santos Bastos, ${ }^{\dagger}$ Sabrina B. Rodriguez Reartes, ${ }^{\S, \perp}$ Marcelo S. Zabaloy, ${ }^{\S, \perp \odot}$ Rafael F. Cassaro,, Reinaldo C. Bazito," Gustavo R. Borges,

${ }^{\dagger}$ Postgraduate Programme in Process Engineering (PEP)/ Postgraduate Programme in Industrial Biotechnology (PBI), Tiradentes University (UNIT), Av. Murilo Dantas, 300, Aracaju, SE Brazil, CEP 49032-490

${ }^{\ddagger}$ Center for Study on Colloidal Systems (NUESC)/Institute of Technology and Research (ITP), Av. Murilo Dantas, 300, Aracaju, SE Brazil, 49032-490

${ }^{\S}$ Planta Piloto de Ingeniería Química - PLAPIQUI (UNS-CONICET), Camino "La Carrindanga” Km 7 - CP $8000 \mathrm{~B}$ - Bahía Blanca, Argentina

"Institute of Chemistry, Department of Fundamental Chemistry, University of São Paulo, São Paulo, SP 05508-000, Brazil

${ }^{\perp}$ Departamento de Ingeniería Química, Universidad Nacional del Sur (UNS)- Bahía Blanca, Argentina

Supporting Information
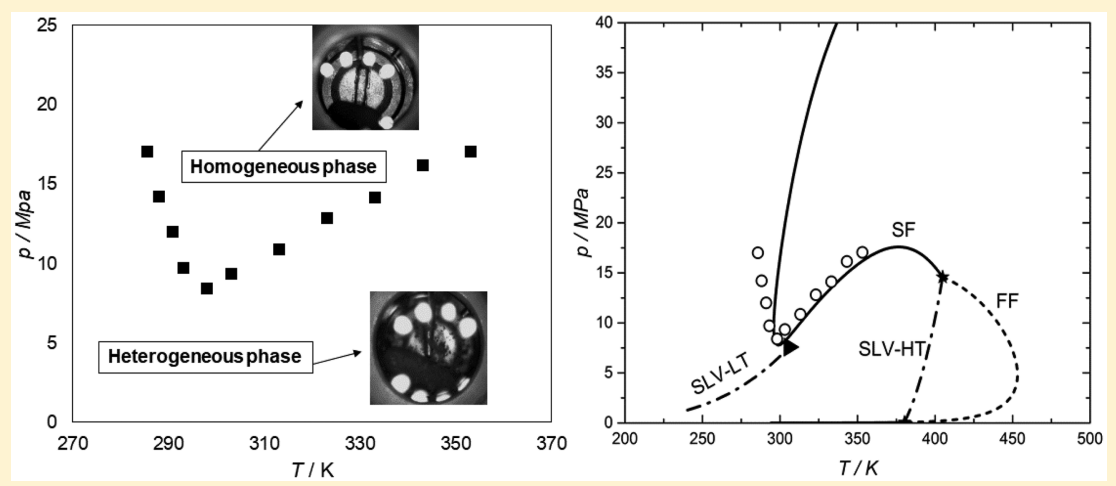

ABSTRACT: The experimental study and thermodynamic modeling of the phase behavior of pressurized reactional systems allows the optimization of several unit operations involved in the process of product formation. In this work, experimental data of phase equilibria for the $\mathrm{CO}_{2}+$ p-nitrobenzaldheyde binary system were obtained through the static synthetic method. The range of temperature, pressure, and p-nitrobenzaldehyde molar fraction investigated were $281-353 \mathrm{~K}, 6.5-25.0 \mathrm{MPa}$, and 2.638 $\times 10^{-3}$ to $5.903 \times 10^{-3}$, respectively. A model previously developed to describe asymmetric mixtures presenting fluid and solid phases was applied to describe the phase behavior of the system. This model uses the Peng-Robinson equation of state (PR$\mathrm{EoS}$ ) to describe the properties of the fluid phases and an expression for the fugacity of p-nitrobenzaldehyde as a pure solid for the solid phase. Different model parametrization strategies were studied, and complete isopleths were calculated considering the fluid-fluid, solid-fluid, and solid-fluid-fluid phase equilibria over wide ranges of temperature and pressure. The experimental results showed nonmonotonic (local minimum) solid-fluid phase behavior for all mixture compositions investigated. The model employed and the parametrization strategies were able to describe the experimentally observed phase behavior.

\section{INTRODUCTION}

The study of asymmetric aldol reactions has grown in the last decades, making this type of reaction one of the most investigated in organic synthesis. ${ }^{1-3}$ They are an important tool to make new carbon-carbon bonds and to control the stereochemistry of the stereogenic centers generated. In a simplified way, the aldol reactions are based on joining two relatively simple molecules to give rise to a more complex one, that is, the $\beta$-hydroxy carbonylated compounds (aldol compounds). ${ }^{3,4}$ These compounds can be used in different areas such as pharmaceutical, cosmetic, flavors, and additives industries. $^{5-8}$
Aldol reactions are generally conducted in a reactional media in which the commonly employed solvents are dimethylformamide (DMF) or dimethyl sulfoxide (DMSO), which are toxic and unsafe due to their high volatility and flammability. ${ }^{4}$ In the synthesis, processing and product quality control of fine chemicals when the compounds are biologically active, it is

Special Issue: Latin America

Received: November 12, 2018

Accepted: February 22, 2019

Published: March 11, 2019 
imperative to take toxicological and ecological issues into account. ${ }^{9,10}$ Thus, the increasing demand for ecological and sustainable processes has resulted in numerous studies and works that aim to replace these kinds of solvents by alternative ones. $^{11}$

The main challenge in this field is to generate products with high efficiency and high enantioselectivity but at low cost and under mild reaction and separation conditions. ${ }^{12}$ Therefore, the proper choice of the solvent is of fundamental importance since the reaction media will strongly affect the enantioselectivity of the product and the reaction yield. ${ }^{13}$

In this sense, the use of sub- or supercritical fluids as pressurized reactional media is an attractive choice that allows a more sustainable process and includes the possibility of tuning the process variables that control the reaction. Through the change in pressure and temperature of the system, the physicochemical properties of the reactional media are modified thus tuning the solubilization capacity, increasing the mass transfer rate and reducing undesirable secondary reactions. ${ }^{8,14-17}$

Within the range of compounds that are employed as solvents in reactions at high pressure, the use of supercritical carbon dioxide is of great interest. ${ }^{4,13,15,16,18-20}$ This is due to some characteristics of $\mathrm{CO}_{2}$ as its low cost and easy separation after a reaction, and to the possibility of controlling several physicochemical properties of this solvent by adjusting the solvent temperature and pressure. ${ }^{21}$

To conduct chemical reactions at high pressure, experimental and theoretical studies of the phase behavior of the system are of major importance, as they give insights on the selection of optimal conditions to perform a chemical reaction at high pressures, allowing the control of the reaction yield and selectivity. Furthermore, through the knowledge of the phase behavior it is possible to optimize the unit operations involved in the product formation, allowing also to establish specifications to manufacture of equipment. ${ }^{8,22}$

The reaction between p-nitrobenzaldehyde and acetone, catalyzed by L-proline is considered as a "model aldolic reaction" in the literature. ${ }^{4,14}$ Considering the possibility of conducting this reaction in a pressurized $\mathrm{CO}_{2}$ reactional media, in this work we study the phase behavior of the $\mathrm{CO}_{2}+\mathrm{p}$ nitrobenzaldehyde (4-nitrobenzaldehyde) binary system.

The experimental measurement is considered the only suitable method for the accurate determination of phase behavior at high pressures. ${ }^{23,24}$ The accuracy and care in conducting experimental phase equilibria measurements, to produce good quality data, allow the fit of thermodynamic models, which may in turn be used to make predictions at conditions different from those observed experimentally, at the laboratory.

In this sense, the objective of this work was the experimental and modeling study of the phase behavior at high pressure of the binary system $\mathrm{CO}_{2}+\mathrm{p}$-nitrobenzaldehyde (4-nitrobenzaldehyde). For carrying out the experimental measurements of phase behavior, the static synthetic method was employed either in isothermal mode or in isobaric mode. Thermodynamic modeling was performed by employing the Peng-Robinson equation of state (PR-EoS) to represent the fluid phases and to model the solid phase by using an expression for the fugacity of the solute as a pure solid. Different strategies for the parametrization of the thermodynamic model were considered, and calculations of complete isopleths were performed considering the fluid-fluid equili- brium, the solid-fluid equilibrium and the solid-fluid-fluid equilibrium, in wide ranges of temperature and pressure.

\section{MATERIAL AND METHODS}

2.1. Chemicals. The chemicals employed in the experimental measurements of high-pressure phase behavior were 4nitrobenzaledhyde and carbon dioxide. For the validation of the experimental apparatus of phase equilibria measurements, ethanol was used. These chemicals were used without any further purification and their specifications are presented in Table 1.

Table 1. Specifications of the Chemicals Used in This Work

\begin{tabular}{llc}
\multicolumn{1}{c}{ chemical name } & \multicolumn{1}{c}{ supplier } & mass fraction purity \\
carbon dioxide & White Martins Praxair Inc. & 0.999 \\
4-nitrobenzaldehyde & Sigma-Aldrich & 0.980 \\
ethanol & Vetec Fine Chemistry Ltd. & 0.995 \\
\hline
\end{tabular}

2.2. Apparatus and Experimental Procedure for Phase Behavior Measurement. The experimental apparatus and procedure were based on the static synthetic method, whose use is widely documented in the literature. ${ }^{25-29} \mathrm{~A}$ diagram of the experimental apparatus used in this work for phase equilibrium measurements is shown in Figure 1.

Briefly, the experimental apparatus consists of a view cell with variable volume made of stainless steel with a maximum volume of $25 \mathrm{~mL}$. The cell has a frontal sapphire window and a lateral sapphire window for visualization of phase transitions and lighting, respectively. A syringe pump (Teledyne ISCO, $260 \mathrm{D}$ ) is used to accurately deliver $\mathrm{CO}_{2}$ into the equilibrium cell and to move the piston inside the cell for pressurization and/or depressurization of the system, using $\mathrm{CO}_{2}$ as pneumatic fluid. Two thermostatic baths (JULABO, AC-F12-EH) were used, respectively, to control the temperature inside the equilibrium cell and to maintain the temperature in the syringe pump reservoir. To monitor the pressure of the system, a pressure transducer (Huba Control, model 5436) was employed. Temperature inside the equilibrium cell was monitored by a type $\mathrm{J}$ thermocouple connected to a temperature indicator. A set of ball valves (Swagelok, $5583 \mathrm{KF} 2$ ) was used to allow or to block the $\mathrm{CO}_{2}$ flow through the tubing of the experimental unit. To control the $\mathrm{CO}_{2}$ flow into the cell and to perform the total depressurization of the experimental unit a set of needle valves (HIP, 1511AF1) was used. To promote the solubilization of the mixture inside the cell a magnetic stirrer was employed together with a magnetic bar.

To favor the visualization of the phase transitions of the studied system, a digital microscope (DINO-LITE, model Dina capture 2.0) was located into the frontal sapphire window of the equilibrium cell and connected to a computer for image acquisition.

The experimental procedure for the phase behavior measurements of the $\mathrm{CO}_{2}+$ p-nitrobenzaldehyde system starts by the weighing of a given mass of solute in an analytical balance (precision of $10^{-4} \mathrm{~g}$ ). This is followed by its introduction into the equilibrium cell. After this, the cell is closed and connected to the system and a known volume of $\mathrm{CO}_{2}$ is introduced into the cell with the aid of the syringe pump, at temperature and pressure of $290 \mathrm{~K}$ and $15.0 \mathrm{MPa}$, respectively. These are the $\mathrm{CO}_{2}$ conditions within the syringe pump during the pumping process and imply a convenient 


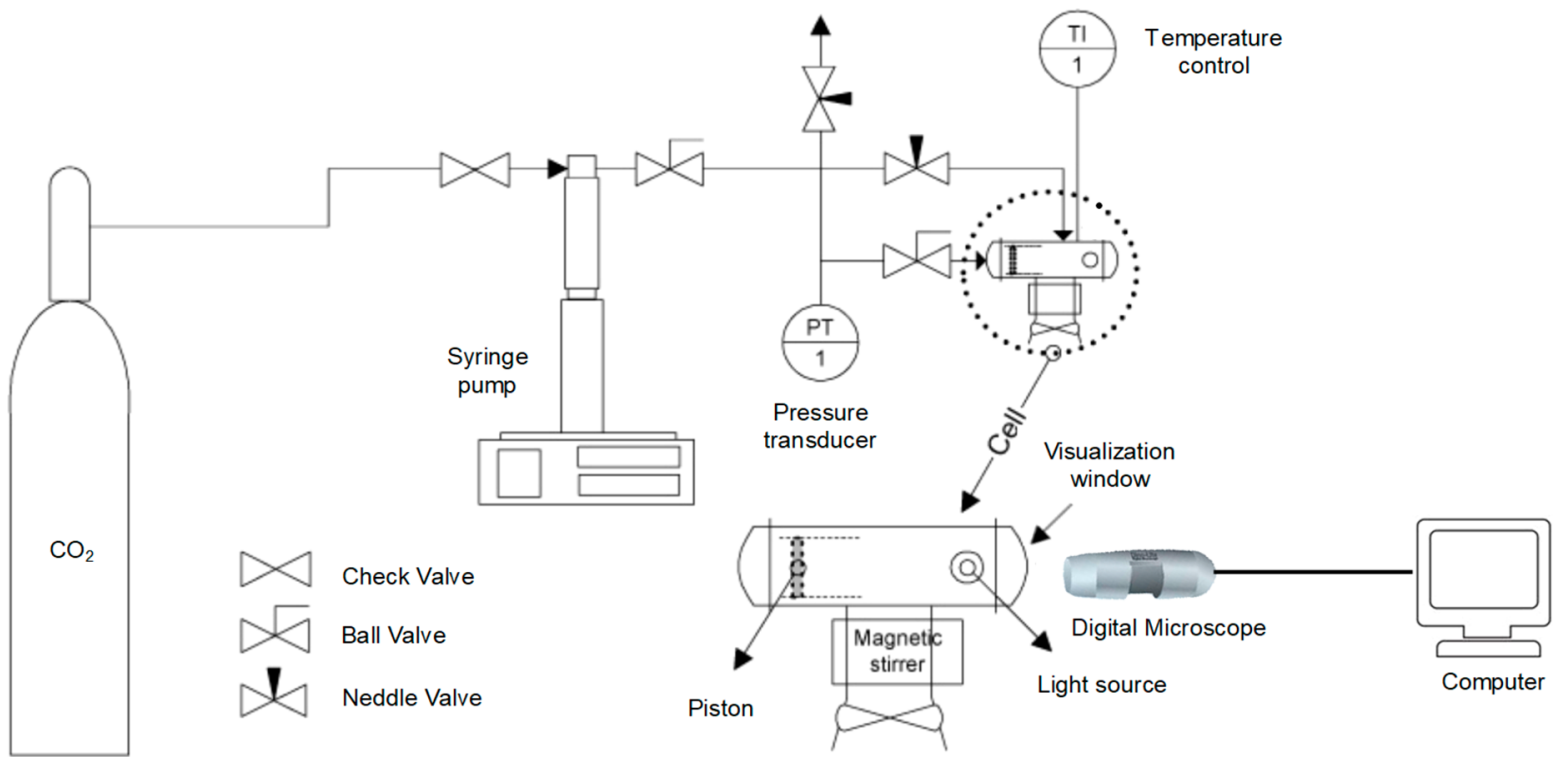

Figure 1. Diagram of the phase equilibria experimental apparatus used in this work.

value for the pure $\mathrm{CO}_{2}$ density. Experimental density values of $\mathrm{CO}_{2}$ needed for quantifying the amount of $\mathrm{CO}_{2}$ loaded into the equilibrium cell and hence quantifying its global concentration can be found in the literature at this temperature and pressure. ${ }^{30}$ Phase transitions were obtained by applying either the isothermal method or the isobaric method, depending on the temperature condition.

In the isothermal method, once the desired temperature was set with constant agitation, the equilibrium cell containing the mixture was pressurized until a single phase was observed. The system was maintained at these conditions for at least $30 \mathrm{~min}$. After this, the system was slowly depressurized at a controlled rate until the appearance of a new phase was detected by visual observation. In this moment, pressure and phase transition type were recorded and the system was repressurized to attain again a single phase condition. The measurement procedure was repeated at least 3 times for each temperature measured.

In the isobaric method, the phase equilibrium experimental data were obtained for different pressure values. In this method, pressure was maintained constant in a specified value and the temperature was increased until the total solubilization of the system was observed. When the system was stable and in a single phase, temperature was slowly decreased at a constant rate until the visual observation of phase transition. Here, the temperature and phase transition type were recorded. After this, the system was heated until it became homogeneous again (all solid dissolved), and it was set to remain under these conditions for at least $30 \mathrm{~min}$, before imposing a new decrease in temperature to obtain another phase transition. As in the isothermal method, this procedure was repeated at least 3 times for each pressure.

The transition points, obtained in the isothermal and isobaric methods adopted here, were determined by visual observation of the first solute solid particles precipitated. The visualization of the solid phase formation was greatly improved with the aim of the digital microscope coupled on the outer wall of the frontal sapphire window of the equilibrium cell.

For all investigated systems, the isobaric method was used when it was verified that a small change in temperature caused a drastic change in the solid-fluid transition pressure, that is, when the slope of the transition curve in the $P-T$ diagrams was found to be high.

With the aim of validating the experimental apparatus for phase behavior measurements and of verifying their reproducibility, some experiments of vapor-liquid equilibria were made before and after the complete study for the system $\mathrm{CO}_{2}(1)+$ p-nitrobenzaldehyde(2) and are presented in Table 2 and Figure 2. The selected system for these tests was $\mathrm{CO}_{2}+$

Table 2. Experimental Phase Equilibrium Data of Temperature $(T)$, Pressure $(P)$ and $\mathrm{CO}_{2}$ Mole Fraction $\left(x \mathrm{CO}_{2}\right)$ for the Binary System $\mathrm{CO}_{2}+$ Ethanol $^{a}$ (This Work)

\begin{tabular}{|c|c|c|c|c|c|}
\hline \multicolumn{3}{|c|}{$T=313.15 \mathrm{~K}$} & \multicolumn{3}{|c|}{$T=333.15 \mathrm{~K}$} \\
\hline$x \mathrm{CO}_{2}$ & $\mathrm{P} / \mathrm{MPa}$ & transition & $x \mathrm{CO}_{2}$ & $\mathrm{P} / \mathrm{MPa}$ & transition \\
\hline \multicolumn{6}{|c|}{ Before Solid-Fluid Measurements } \\
\hline 0.207 & 3.15 & $\mathrm{VLE}^{b}$ & 0.207 & 3.96 & VLE \\
\hline 0.411 & 6.11 & VLE & 0.411 & 8.24 & VLE \\
\hline 0.611 & 7.65 & VLE & 0.611 & 9.70 & VLE \\
\hline 0.807 & 7.76 & VLE & 0.807 & 11.10 & VLE \\
\hline \multicolumn{6}{|c|}{ After Solid-Fluid Measurements } \\
\hline 0.414 & 6.44 & VLE & 0.414 & 8.01 & VLE \\
\hline 0.605 & 7.38 & VLE & 0.605 & 9.52 & VLE \\
\hline
\end{tabular}

$a_{u(T)}=1.3 \mathrm{~K}, u\left(x \mathrm{CO}_{2}\right)=0.001$, and $u(p)=0.17 \mathrm{MPa}$. $u$ is the standard uncertainty in the experimental data. ${ }^{b}$ VLE: vapor-liquid equilibrium.

ethanol and the results were compared with literature experimental data. ${ }^{31-34}$ For the $\mathrm{CO}_{2}+$ ethanol system, the molar fractions of $\mathrm{CO}_{2}$ experimentally set were $0.207,0.411$, 0.589 and 0.793 , and the set temperatures were 313.15 and $333.15 \mathrm{~K}$.

For the $\mathrm{CO}_{2}(1)+$ p-nitrobenzaldehyde(2) system, the set molar fractions of the solute in $\mathrm{CO}_{2}\left(2.638 \times 10^{-3}, 3.525 \times\right.$ $10^{-3}, 4.415 \times 10^{-3}$, and $\left.5.903 \times 10^{-3}\right)$ have values that were selected based on the previously considered concentrations of this compound in the model aldol reaction of p-nitrobenzaldehyde + acetone. $^{18}$ Experiments at higher molar fraction of p-nitrobenzaldehyde were not carried out in this work due to the maximum operating pressure limit of the 

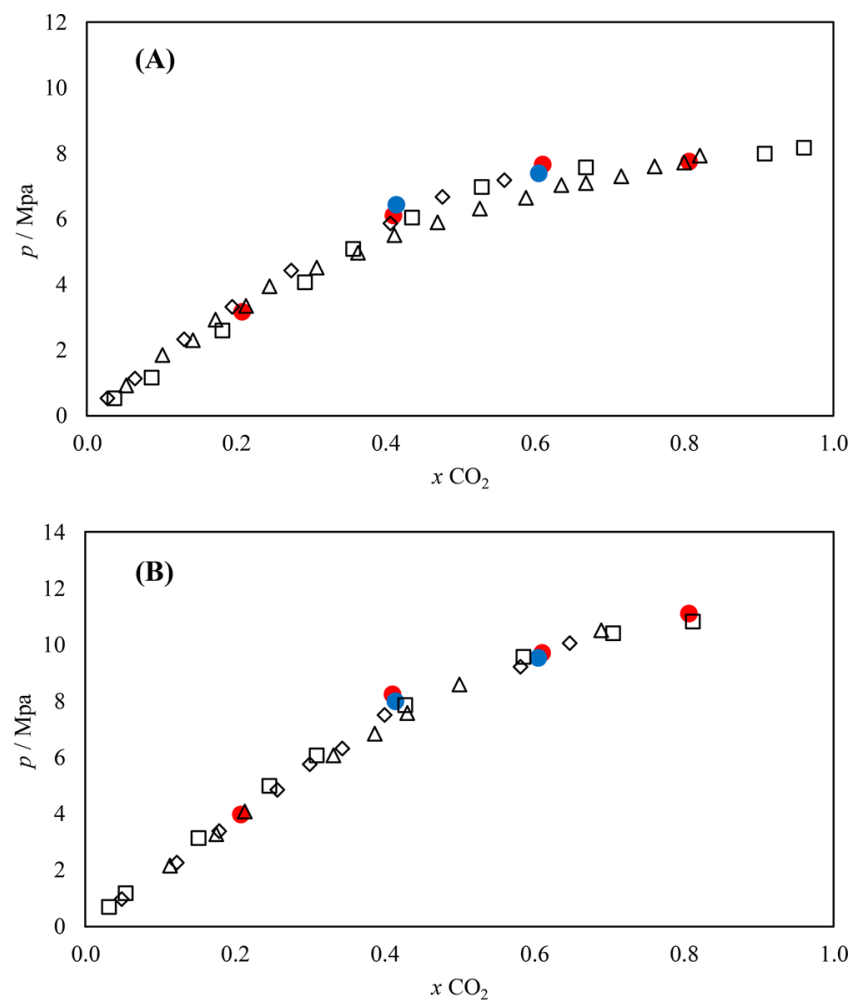

Figure 2. Experimental bubble pressure versus liquid phase $\mathrm{CO}_{2}$ mole fraction for the system $\mathrm{CO}_{2}+$ ethanol. Comparison of data obtained in this work (red and blue full symbols) with data from literature (empty symbols). (A) This work at $313.15 \mathrm{~K}$ before p-nitrobenzaldehyde study (red-filled circle); this work at $313.15 \mathrm{~K}$ after $\mathrm{p}$ nitrobenzaldehyde study (blue-filled circle); SUZUKY et al ${ }^{31}$ at 313.4 $\mathrm{K}(\square)$; DAY et al. ${ }^{32}$ at $313.14 \mathrm{~K}(\triangle)$; SECUIANU et al. ${ }^{33}$ at 313.15 $\mathrm{K}(\diamond)$. (B) This work at $333.15 \mathrm{~K}$ before p-nitrobenzaldehyde study (red-filled circle); this work at $333.15 \mathrm{~K}$ after p-nitrobenzaldehyde study (blue-filled circle); SUZUKY et al. ${ }^{31}$ at $333.4 \mathrm{~K}(\square)$; SECUIANU et al. ${ }^{33}$ at $333.15 \mathrm{~K}(\diamond)$; SIMA et al. ${ }^{34}$ at $333.2 \mathrm{~K}(\triangle)$.

experimental apparatus. Temperature range investigated was from 281 to $353 \mathrm{~K}$ and the pressure range was up to $25.0 \mathrm{MPa}$. The upper values of temperature and pressure ranges were selected due to the maximum operating conditions of the experimental apparatus. The lower value of temperature of the range investigated $(281 \mathrm{~K})$ was set to verify the sign of the pressure versus temperature slope of the experimental curve of solid-fluid phase transition. Results for the $\mathrm{CO}_{2}(1)+\mathrm{p}$ nitrobenzaldehyde(2) system are presented in Table 3 and Figure 3.

2.3. Modeling of the Phase Behavior of the $\mathrm{CO}_{2}(1)+$ p-Nitrobenzaldehyde(2) System. In this work, we modeled the phase behavior of the system carbon dioxide $(1)+\mathrm{p}$ nitrobenzaldehyde(2). We present calculated isopleth phase envelopes made of fluid-fluid (FF) and solid-fluid (SF) equilibrium segments. We also show computed solid-fluidfluid (SFF) equilibrium segments which contribute to the heterogeneous regions of the isopleths. We used the experimental data obtained in this work to fit the model parameters. The main aim is to establish whether it would be possible for the model to capture the minima in pressure experimentally observed for the SF equilibria at constant overall composition.

It is not easy to establish a priori whether two components would be partially or completely miscible in the solid phase.
Table 3. Experimental Solid-Fluid Equilibrium Data of Temperature $(T)$, Pressure $(P)$ and 4-Nitrobenzaldehyde Mole Fraction $\left(x_{2}\right)$ for the Binary System $\mathrm{CO}_{2}(1)+\mathrm{p}$ Nitrobenzaldehyde $(2)^{a}$ (This Work)

\begin{tabular}{|c|c|c|c|c|c|}
\hline \multicolumn{3}{|c|}{$x_{2}=2.638 \times 10^{-3}$} & \multicolumn{3}{|c|}{$x_{2}=4.415 \times 10^{-3}$} \\
\hline$T / K$ & $P / \mathrm{MPa}$ & method $^{b}$ & $T / K$ & $\mathrm{P} / \mathrm{MPa}$ & method $^{b}$ \\
\hline 281.05 & 11.50 & IB & 290.65 & 20.00 & $\mathrm{ib}$ \\
\hline 289.25 & 7.50 & IB & 294.45 & 14.00 & ib \\
\hline 298.15 & 6.73 & IT & 298.15 & 12.01 & IT \\
\hline 303.15 & 7.61 & IT & 303.15 & 10.78 & IT \\
\hline 308.15 & 8.28 & IT & 308.15 & 11.68 & IT \\
\hline 313.15 & 9.16 & IT & 313.15 & 12.59 & IT \\
\hline 323.15 & 10.98 & IT & 323.15 & 13.97 & IT \\
\hline 333.15 & 12.80 & IT & 333.15 & 15.23 & IT \\
\hline \multicolumn{3}{|c|}{$x_{2}=3.525 \times 10^{-3}$} & \multicolumn{3}{|c|}{$x_{2}=5.903 \times 10^{-3}$} \\
\hline$T / \mathrm{K}$ & $\mathrm{P} / \mathrm{MPa}$ & method $^{b}$ & $T / \mathrm{K}$ & $\mathrm{P} / \mathrm{MPa}$ & method $^{b}$ \\
\hline 285.75 & 17.00 & IB & 298.05 & 24.50 & IB \\
\hline 288.15 & 14.20 & IB & 305.45 & 21.00 & IB \\
\hline 291.05 & 12.00 & IB & 313.15 & 17.48 & IT \\
\hline 293.15 & 9.70 & IT & 318.15 & 16.55 & IT \\
\hline 298.15 & 8.39 & IT & 323.15 & 15.56 & IT \\
\hline 303.15 & 9.33 & IT & 333.15 & 16.49 & IT \\
\hline 313.15 & 10.85 & IT & 343.15 & 17.65 & IT \\
\hline 323.15 & 12.81 & IT & & & \\
\hline 333.15 & 14.11 & IT & & & \\
\hline 343.15 & 16.14 & IT & & & \\
\hline 353.15 & 17.05 & IT & & & \\
\hline
\end{tabular}

${ }^{a} u(T)=1.3 \mathrm{~K}, u\left(x_{2}\right)=1.0 \times 10^{-5}$, and $u(p)=0.17 \mathrm{MPa} . u$ is the standard uncertainty in the experimental data. ${ }^{b}$ Method used for the phase equilibria determination. IT $=$ isothermal method; $\mathrm{IB}=$ isobaric method.

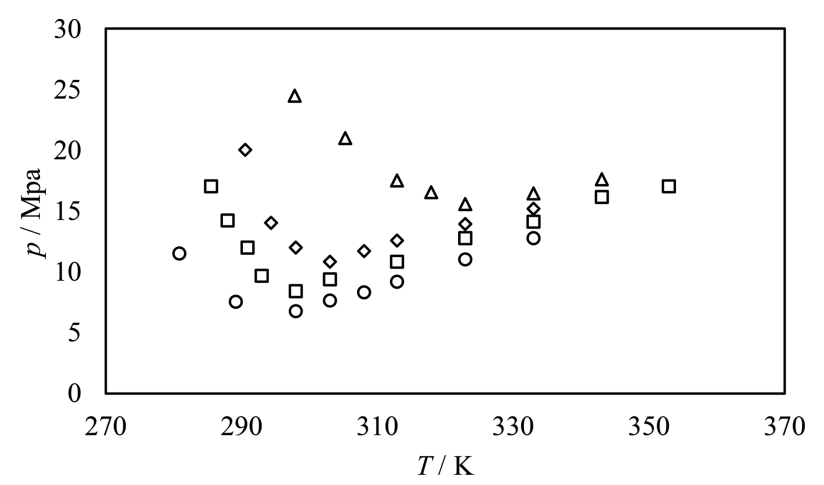

Figure 3. Experimental solid-fluid equilibrium pressure-temperature plots for the system $\mathrm{CO}_{2}(1)+$ p-nitrobenzaldehyde $(2)$ at several fluid phase molar fractions of p-nitrobenzaldehyde: $(\bigcirc) 2.638 \times 10^{-3},(\square)$ $3.525 \times 10^{-3},(\diamond) 4.415 \times 10^{-3}$, and $(\Delta) 5.903 \times 10^{-3}$. See Table 3 .

Solid phase miscibility is considered negligible for nonpolar compounds with important differences in molecular size and shape. ${ }^{46}$ However, similarity in molecular size and shape does not guarantee full miscibility in solid phase, as it becomes evident from comparing the experimental phase behavior of the systems fluorene/dibenzofuran and fluorene/dibenzothiophene $^{46}$.

On the basis of practical experience, different rules of thumb have been followed to predict the occurrence of solubility in solid state. ${ }^{47}$ However, the current knowledge seems insufficient to merely predict the prevalence of solid solutions over solid precipitation in (practically) pure state, or vice versa, 
Table 4. Critical Temperature $\left(T_{\text {crit }}\right)$, Critical Pressure $\left(P_{\text {crit }}\right)$, and Acentric Factor $(\omega)$ of Pure Compounds and Binary Interaction Parameters $\left(k_{12}\right.$ and $\left.l_{12}\right)$ for the PR-EoS ${ }^{37}$

\begin{tabular}{|c|c|c|c|c|c|}
\hline compound/System & $T_{\text {crit }} / \mathrm{K}$ & $P_{\text {crit }} / \mathrm{MPa}$ & $\omega$ & $k_{12}$ & $l_{12}$ \\
\hline $\mathrm{CO}_{2}(1)$ & $304.21^{a}$ & $7.383^{a}$ & $0.22362^{a}$ & & \\
\hline p-nitrobenzaldehyde(2) & $782.45^{b}$ & $4.6306^{c}$ & $0.638^{b}$ & & \\
\hline $\mathrm{CO}_{2}(1)+$ p-nitrobenzaldehyde $(2)$ & & & & -0.32732 & \\
\hline $\mathrm{CO}_{2}(1)+$ p-nitrobenzaldehyde $(2)$ & & & & & -0.85921 \\
\hline
\end{tabular}

without any additional experimental solid-state information for the studied system.

As the simplest possible approach, we have assumed in this work that the solid phase is only formed by pure pnitrobenzaldehyde, that is, we assume total absence of miscibility in solid state, which seems reasonable in view of the important differences in molecular size and shape between p-nitrobenzaldehyde and $\mathrm{CO}_{2}$. Besides, according to ref 46, between the two extreme assumptions for the solid state, that is, complete miscibility versus complete immiscibility, it is in most cases more realistic to consider complete immiscibility.

Despite our decision of imposing precipitation in pure state for modeling purposes, we do have not enough evidence for fully ruling out the possibility of having some $\mathrm{CO}_{2}$ coexisting with the p-nitrobenzaldehyde at equilibrium in the solid phase under high enough pressure.

The modeling approach used in this work was previously applied to the representation of the phase behavior of binary asymmetric mixtures where it can be assumed that the solid phase is made of the pure heavy compound. ${ }^{25,35,36}$ As in the quoted references, we used here PR-EoS ${ }^{37}$ for calculating component fugacities in fluid state for pure compounds and mixtures. For solid-fluid and solid-fluid-fluid equilibrium calculations, we assumed that the solid phase is made of pure p-nitrobenzaldehyde(2) since its mixture with $\mathrm{CO}_{2}(1)$ is highly asymmetric. "High asymmetry" means that this binary system presents important differences in molecular size and shape. The fugacity of the pure heavy compound in solid state $f_{2}^{S}\left(T, P, v_{\mathrm{o}}\right)$ at system temperature $(T)$ and pressure $(P)$ is given by eq 1

$$
f_{2}^{S}\left(T, P, v_{\mathrm{o}}\right)=\hat{f}_{2}\left(T, 1, v_{\mathrm{o}}\right) \exp (U)
$$

In eq $1, v_{\mathrm{o}}$ is the molar volume of the pure heavy component (component " 2 "), in (subcooled hypothetical) liquid state, at $T$ and $P$. Such pure liquid has a fugacity $\hat{f}_{2}\left(T, 1, v_{\mathrm{o}}\right)$. Both, $v_{\mathrm{o}}$ and $\hat{f}_{2}\left(T, 1, v_{\mathrm{o}}\right)$ are given in this work by the PR-EoS. ${ }^{37^{\circ}}$ The exponential factor in eq 1 relates [through eq 1] the hypothetical liquid state to the solid state for a pure substance at given temperature and pressure. The variable $U$, which depends on $T$ and $P$, is defined as follows

$$
\begin{aligned}
U= & \frac{\Delta v^{S-\mathrm{L}}}{R T_{\text {tp }}}\left[C_{1}\left(1-\frac{T_{\mathrm{tp}}}{T}\right)+C_{2}\left(\frac{T_{\mathrm{tp}}}{T}-1+\ln \left(\frac{T}{T_{\mathrm{tp}}}\right)\right)\right. \\
& \left.+C_{3}\left(\frac{T}{2 T_{\mathrm{tp}}}-1+\frac{T_{\text {tp }}}{2 T}\right)+\frac{T_{\text {tp }}}{T}\left(P-P_{\mathrm{tp}}\right)\right]
\end{aligned}
$$

In eq 2 , the constants $T_{\mathrm{tp}}, P_{\mathrm{tp}}, \Delta v^{\mathrm{S}-\mathrm{L}}, C_{1}, C_{2}$, and $C_{3}$ correspond to the pure heavy component (component " 2 "). $\Delta v^{\mathrm{S}-\mathrm{L}}$ is the solid-liquid molar volume difference $\left(v_{\text {solid }}-\right.$ $\left.v_{\text {liquid }}\right) . T_{\mathrm{tp}}$ and $P_{\mathrm{tp}}$ are the triple point temperature and pressure, respectively; and $R$ is the universal gas constant. The constants $C_{1}, C_{2}$, and $C_{3}$ characterize (together with $T_{\text {tp }}$ and $\left.P_{\text {tp }}\right)$ the pure heavy component solid-liquid equilibrium curve ( $P$ versus $T$ melting curve). More details about eqs 1 and 2, and about the model used here, are given in literature. ${ }^{25,35,36}$ The system of equations to be solved when performing the phase equilibrium calculations arises from imposing the classical necessary conditions, that is, equal temperatures, equal pressures, and equal fugacities of each component in all phases. More details about the system of equations of a SF equilibrium point are given in Supporting Information. With regard to the calculation algorithms, we used numerical continuation methods (NCMs) for computing all phase equilibrium curves. ${ }^{36,38,39}$

2.3.1. Parametrization. The critical properties and acentric factor of $\mathrm{CO}_{2}(1)$ are shown in Table 4. They were taken from the DIPPR database. ${ }^{40}$ For p-nitrobenzaldehyde(2), we estimated the critical temperature and the acentric factor through the method of Hukkerikar et al. ${ }^{41}$ We selected this method since it constitutes a revised and improved groupcontribution $^{+}\left(\mathrm{GC}^{+}\right)$model employed for the estimation of pure component properties. $\mathrm{GC}^{+}$means "combined groupcontribution (GC) method and atom connectivity index (CI) method". For the parameter determination of this $\mathrm{GC}^{+}$method large experimental data sets of pure component properties were considered ${ }^{41}$ including hydrocarbons, oxygenated compounds, nitrogenated compounds, polyfunctional compounds, and so forth. This $\mathrm{GC}^{+}$method $^{41}$ accounts for the contributions of first, second, and third order groups to estimate the properties of the pure compounds.

The critical pressure of p-nitrobenzaldehyde(2) was adjusted to make the PR-EoS give subcritical liquid densities in the order of the triple point liquid density. Such p-nitrobenzaldehyde(2) triple point liquid density can be estimated using the Goodman et al. formula. ${ }^{42}$ This formula relates the solid density of a pure compound at temperature $T$ with the liquid density of such compound at a given temperature $T_{\mathrm{t}}$. In our case, we used the solid density of pure p-nitrobenzaldehyde at $298.15 \mathrm{~K}$ (taken from ref 43) in the Goodman et al. formula ${ }^{42}$ to estimate the pure p-nitrobenzaldehyde liquid density at the triple point. The values obtained for the pure p-nitrobenzaldehyde(2) critical constants and acentric factor $(\omega)$ are reported in Table 4 . The pnitrobenzaldehyde(2) triple point temperature $T_{\text {tp }}$ is in this work the one reported by Yaws. ${ }^{43}$ For consistency, the pnitrobenzaldehyde(2) triple point pressure $P_{\text {tp }}$ was set equal to the PR-EoS vapor-liquid equilibrium pressure computed at $T_{\text {tp. }}$.

The values for the parameters $k_{12}, l_{12}, \Delta v^{\mathrm{S}-\mathrm{L}}, C_{1}, C_{2}$, and $C_{3}$ need still to be set. $k_{12}$ and $l_{12}$ are the attractive and the repulsive interaction parameters, respectively, which appear in the quadratic mixing rules of the PR-EoS. The only information related to the p-nitrobenzaldehyde(2) fusion curve that we have found in the literature is just the triple 

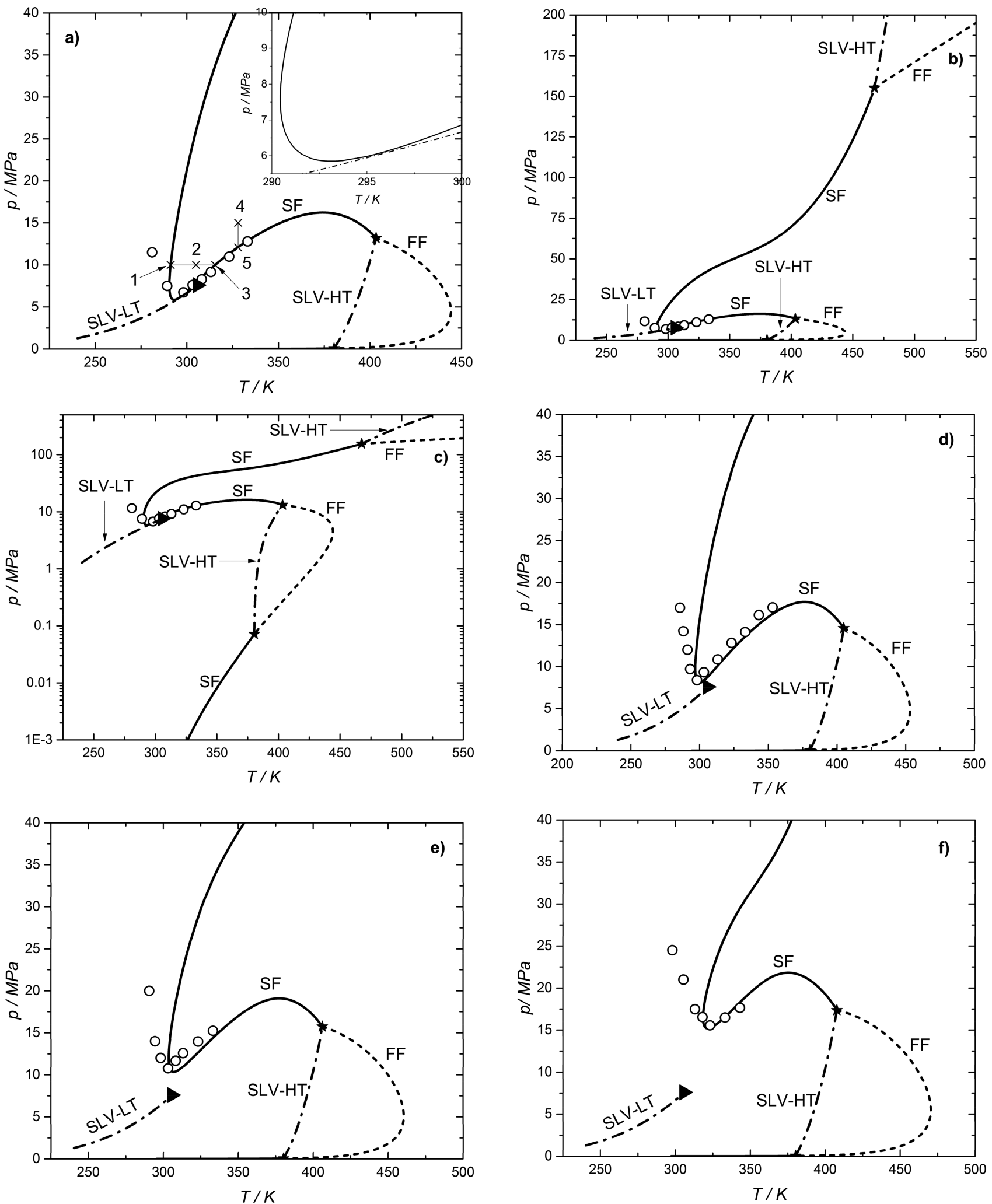

Figure 4. Calculated Isopleths for the $\mathrm{CO}_{2}(1)+$ p-nitrobenzaldehyde(2) mixture. Fluid phase p-nitrobenzaldehyde mole fraction values: (a) 2.638 $\times 10^{-3}$, (b) $2.638 \times 10^{-3}$ [as panel a but in a wider pressure range], (c) $2.638 \times 10^{-3}$ [as panel b but with pressure in log scale], (d) $3.525 \times 10^{-3}$, (e) $4.415 \times 10^{-3}$, (f) $5.903 \times 10^{-3}$. O: experimental solid-fluid equilibrium data (this work, Table 3). Phase equilibria calculated using the PR-EoS and eq 1: solid lines, solid-fluid (SF); dashed lines, fluid-fluid (FF); dashed-dotted lines and stars, solid-liquid-vapor (SLV-LT and SLV-HT;); 1 st critical end point. Model parameters from Tables 4 and 5.

point temperature previously mentioned, that is, we have not found in the literature experimental data for the fusion curve of pure p-nitrobenzaldehyde(2) in a wide range of pressure. Such data would have been useful to obtain the values of parameters $C_{1}, C_{2}$, and $C_{3}$. It seems that neither are there experimental $\mathrm{FF}$ equilibrium data for $\mathrm{CO}_{2}(1)+$ p-nitrobenzaldehyde(2) 
available in the literature, which would be of use in estimating parameters $k_{12}$ and $l_{12}$. In view of the lack of binary FF experimental data and after some preliminary work, we set the $k_{12}$ and $l_{12}$ parameters at values (Table 4) that assured an appropriate relative stability between the calculated fluid-fluid and solid-fluid equilibria. Next, we estimated the values for $\Delta v^{\mathrm{S}-\mathrm{L}}, C_{1}, C_{2}$, and $C_{3}$ from four selected SF experimental data. We did so by solving the system of equations that arises from imposing, simultaneously for the four selected SF equilibrium points, the SF isofugacity condition for component 2 at the experimental $T, P$, and fluid phase composition. Several subsets of four SF experimental data were considered.

The final set of parameter values was selected as the one that best reproduced the experimental data while meeting the proper thermodynamic stability constraints. To fix ideas, the parameter values that led to Figure $4 \mathrm{a}$ are such that, for example., a solid-fluid equilibrium calculated at $T$ and $P$ values within the region delimited in Figure $4 a$ by the SLV-HT and FF lines, is thermodynamically unstable with respect to a fluid-fluid equilibrium calculated at the same $T$ and $P$.

The final values for the binary interaction parameters appear in Table 4, while Table 5 presents the parameters for eq 2 for p-nitrobenzaldehyde(2).

Table 5. p-Nitrobenzaldehyde(2) Constants for Equation 2

$\begin{array}{ll}T_{\text {tp }} / K^{a} & 380.15 \\ P_{\text {tp }} / \mathrm{MPa}^{b} & 1.820530 \times 10^{-04} \\ \Delta v^{S-L} /\left(\mathrm{m}^{3} / \mathrm{kmol}\right) & -0.09174286 \\ \mathrm{C}_{1} / \mathrm{MPa} & -122.4207995 \\ \mathrm{C}_{2} / \mathrm{MPa} & 35209.40471 \\ \mathrm{C}_{3} / \mathrm{MPa} & -37599.18297\end{array}$

${ }^{a}$ From Yaws. ${ }^{43}{ }^{b} P_{\text {tp }}=$ PR-EoS ${ }^{37}$ pure compound vapor-liquid equilibrium pressure at the triple point temperature $T_{\mathrm{tp}} . \Delta v^{\mathrm{S}-\mathrm{L}}$ is the solid-liquid molar volume difference.

The values in Table 5 for parameters $T_{\mathrm{tp}}, P_{\mathrm{tp}}, C_{1}, C_{2}$, and $C_{3}$ predict a (not shown) pure p-nitrobenzaldehyde(2) fusion curve for which $P$ is a monotonically increasing function of $T$. This is the typically expected behavior (e.g., water is an exception).

\section{RESULTS AND DISCUSSION}

3.1. Experimental Apparatus Validation and Reproducibility. The validation and reproducibility evaluation of the experimental apparatus used in this work to measure phase equilibrium data was made through measurements for the $\mathrm{CO}_{2}$ + ethanol binary system. This system was chosen because of the availability of high-quality measured data. The experimental data for validation and reproducibility evaluation were measured at 313.15 and $333.15 \mathrm{~K}$ for different $\mathrm{CO}_{2}$ molar fractions and are presented in Table 2. Figure 2 shows the data obtained for the system $\mathrm{CO}_{2}+$ ethanol in this work compared to literature experimental data. ${ }^{31-34}$

As it can be seen in Figure 2, the obtained results show that the experimental apparatus used here is reliable since the data measured in this work for the system $\mathrm{CO}_{2}+$ ethanol are consistent with data from literature for this system. It is also possible to verify the repeatability of the new data from comparing the $\mathrm{CO}_{2}+$ ethanol data measured before (red circles in Figure 2) and after (blue circles in Figure 2) performing the equilibrium study for the system $\mathrm{CO}_{2}+\mathrm{p}$ nitrobenzaldehyde.
3.2. $\mathrm{CO}_{2}(1)+$ p-Nitrobenzaldehyde(2) Experimental Data. The phase behavior of the $\mathrm{CO}_{2}(1)+$ p-nitrobenzaldehyde(2) system was experimentally measured in the temperature range from 281.05 to $353.15 \mathrm{~K}$ and in the pressure range from 6.73 to $25.0 \mathrm{MPa}$. The molar fractions of $\mathrm{p}$ nitrobenzaldehyde studied in this work were $2.638 \times 10^{-3}$, $3.525 \times 10^{-3}, 4.415 \times 10^{-3}$, and $5.903 \times 10^{-3}$.

As presented in Table 3, for all experimental conditions investigated, only solid-fluid (SF) transitions were observed, that is, the p-nitrobenzaldehyde crystallization. The existence of additional liquid-liquid (LL) or vapor-liquid (VL) transitions happening after solid precipitation is not discarded. The observed precipitation of solid particles on the inner surface of the sapphire window (window meant to visualize the cell contents) made impossible to accurately detect any phase transition occurring after an already found solid-fluid transition. As it shown later in this manuscript, the model predicts the existence of solid-liquid-vapor equilibrium lines together with liquid-vapor and solid-fluid regions, all located within the heterogeneous region of constant overall composition $\mathrm{CO}_{2}(1)+$ p-nitrobenzaldehyde(2) equilibrium diagrams. However, the model does not predict stable liquidliquid equilibria.

Isopleths of solid-fluid transitions obtained experimentally for the binary system $\mathrm{CO}_{2}(1)+$ p-nitrobenzaledhyde(2) are shown in Figure 3. In this figure it is possible to assess the influence of the p-nitrobenzaldehyde overall concentration on the phase behavior of this system. By looking at the $P-T$ curves it is possible to observe that, at a fixed temperature, the higher the solute molar fraction, the higher the pressure needed to maintain the system at a single phase. This behavior can be explained by the capacity of solvation of carbon dioxide which, although it does not have a dipole moment, it has a quadrupole moment, thus making it possible to solubilize polar compounds such as p-nitrobenzaldehyde. ${ }^{44}$

Another interesting phenomenon that can be seen in Figure 3 is the change in the behavior within the isopleths for each mixture composition studied. The local minimum in transition pressure for each isopleth is displaced to higher temperatures as the concentration of p-nitrobenzaldehyde in the system increases. To the right of the minimum in each isopleth, the transition pressure increases with increasing temperature. This behavior is reversed when the temperature is less than the temperature of the minimum, that is, the transition pressure increases as temperature decreases.

3.3. Modeling Results. By considering the modeling approach previously outlined and the parameters of Tables 4 and 5 we calculated, for the $\mathrm{CO}_{2}(1)+$ p-nitrobenzaldehyde $(2)$ system, the global phase behavior (which includes critical and three-phase lines), and also a number of isopleths at the experimental global compositions in this work. For the parameters in Tables 4 and 5, the model predicts a phase behavior of type F (see Supporting Information) according to the classification proposed by Yamamoto et al. ${ }^{45}$

In type F (detailed description in Supporting Information), phase behavior there is a critical locus (low temperature (LT) critical line) which originates at the critical point of the light component $\left(\mathrm{CO}_{2}(1)\right)$ and ends at a terminal critical point named "first critical end point" (1st CEP), where a binary critical (fluid) phase is at equilibrium with a solid phase made of the pure heavy component (p-nitrobenzaldehyde(2)). From the "1st CEP", a solid-liquid-vapor (SLV) locus (SLV-LT line) originates and extends toward low temperatures (LT). 
There is also a second (high temperature, HT) critical line that originates at the critical point of the heavy compound ( $p$ nitrobenzaldehyde(2)) and ends at a terminal critical point named "2nd CEP". From this point, a SLV locus (SLV-HT) begins, and ends at low pressure, at the triple point (TP) of the pure heavy compound. For the parameter values in Tables 4 and 5, we observed that the calculated SLV-HT line extends to very high pressures without ever meeting the HT critical line. This is true at least up to a $100 \mathrm{MPa}$ pressure. We also observed that the SLV-HT line and the HT critical line are practically parallel at such high pressures. Thus, the absence of a second CEP may remain at even greater pressures.

We emphasize that in type $\mathrm{F}$ (Supporting Information) behavior there are two SLV curves, that is, the SLV curve of low temperature (SLV-LT) and the SLV curve of high temperature (SLV-HT). There are also two critical lines, the LT critical line and the HT critical line, which have been calculated in this work but are not shown here. A given, here calculated, "phase envelope" is made of FF and SF equilibrium segments. The rest of the isopleth (constant global composition diagram) includes the relevant sections of the SLV curves. Such sections are those that do not violate the mass conservation constraint at the specified global composition. Further details about the type $\mathrm{F}$ phase behavior are given in the Supporting Information of this work.

Figure $4 \mathrm{a}$ shows the isopleth obtained with the model (PREoS and eq 1) and the experimental SF equilibrium data (circles) for the $\mathrm{CO}_{2}(1)+$ p-nitrobenzaldehyde(2) system at overall mole fraction $z_{2}=2.638 \times 10^{-3}$. Figure 4 a shows a calculated SF segment at high pressure (solid line) that presents a local minimum in pressure, like the experimental data. This segment ends at an SLV point (star) from which a segment of bubble points (FF dashed line) arises, then the FF segment reaches a maximum in temperature and ends at another SLV point at low pressure (star). Here, another (low pressure) SF segment originates which can be better seen in Figure $4 c$ due to the log scale in pressure.

The calculated high-pressure SF segment (solid line in Figure $4 a$ ) is quite consistent with the experimental data (empty circles in Figure 4a), that is, the model is roughly able to describe the data capturing the unusual behavior of this system, that is, the minimum in pressure experimentally observed for the SF segment. However, for this particular system, although the model gives a negative SF slope to the left of the minimum pressure point (see the inset in Figure 4a), it does it in a pressure range significantly narrower than that of the experimental data located to the left of the experimental minimum pressure point.

Figure 4a also shows the relevant sections of the calculated three-phase curves (dotted-dashed lines), which describe the heterogeneous zone of the isopleth under study. In particular, it is observed that the complete SLV-LT curve contributes to the heterogeneous region of this isopleth. In Figure 4a, part of the SLV-LT line appears to (but does not) overlap, in the PTprojection, with the high-pressure SF segment of the phase envelope. This is more clearly seen in the Figure $4 \mathrm{a}$ inset. The (not shown) component 2 mole fraction values of the two fluid phases of the SLV-LT locus at any temperature are lower than $2.638 \times 10^{-3}$. Thus, the complete SLV-LT locus contributes to the heterogeneous region of the isopleth in Figure 4a. It is also indicated in Figure 4a the calculated first CEP (full triangle) and the points of intersection of the SLV-HT line with the phase envelope (stars). In a given intersection point (star), one of the fluid phases at SLV equilibrium has a composition equal to the one of the isopleth $\left(z_{2}=2.638 \times 10^{-3}\right)$.

In Figure $4 \mathrm{a}$, the calculation results and the experimental data show that there is a range of pressure in which it is possible to reach at constant pressure, SF transitions from a homogeneous fluid initial state, either by lowering the temperature (normal behavior) or by increasing the temperature (retrograde behavior). The normal behavior of this system is illustrated in Figure 4a when we solubilize the solid by increasing the temperature (at a constant pressure of 10 $\mathrm{MPa}$ ), that is, when going from point 1 (SF phase boundary) to point 2 (homogeneous fluid phase).

The retrograde behavior appears, for instance, when going from point 3 (SF phase boundary) to point 2 (homogeneous fluid phase) (Figure 4a). In this case, the melting (or solubilization) of the solid phase is carried out by lowering the temperature (retrograde fusion at constant pressure). Vice versa, when going from point 2 to point 3 (SF phase boundary) (Figure 4a), a solid phase is produced by increasing the temperature (retrograde solidification at constant pressure). This phenomenon can be attributed to the decrease in density (and consequently in solvent power) for the solvent $\left(\mathrm{CO}_{2}\right)$, when the temperature is increased. ${ }^{35}$

The model also predicts (in correspondence with the experimental data) for this system, the retrograde crystallization of p-nitrobenzaldehyde at constant temperature. For example, at $T=328 \mathrm{~K}$ it is possible to go from a homogeneous fluid state (at $15.0 \mathrm{MPa}$ ), point 4 in Figure $4 \mathrm{a}$, to a SF state when crossing the SF phase boundary (approximately at 12.1 $\mathrm{MPa}$ ), point 5 in Figure $4 \mathrm{a}$. That is, a solid appears due to a decrease in pressure at a constant temperature. At pressures higher than the maximum pressure of Figure $4 \mathrm{a}(40.0 \mathrm{MPa})$, the model predicts a new intersection point (Figure 4b) between the SF line and the SLV-HT line from which a new FF segment that extends toward higher pressures arises (see Figure $4 \mathrm{~b}$ at pressures greater than $150 \mathrm{MPa}$ ). Also, a new segment of the SLV-HT line appears within the isopleth heterogeneous region.

Figures $4 \mathrm{~d}-\mathrm{f}$ present the isopleths for, respectively, $z_{2}=$ $3.525 \times 10^{-3}, z_{2}=4.415 \times 10^{-3}$, and $z_{2}=5.903 \times 10^{-3}$. The analogy with the isopleth of $z_{2}=2.638 \times 10^{-3}$ (Figure 4a) is complete. The analysis of their phase behavior at high pressures and at low pressures is similar to the one performed through, respectively, Figures $4 \mathrm{~b}, \mathrm{c}$ for $z_{2}=2.638 \times 10^{-3}$.

As previously stated, when the p-nitrobenzaldehyde content in the studied mixture increases (from $2.638 \times 10^{-3}$ to $5.903 \times$ $10^{-3}$ in mole fraction), the experimentally observed local minimum in the $P$ versus $T$ solid-fluid curve moves to higher temperatures and pressures. From Figures $4 a, d-f$, we observe that the model properly captures this shift of the minimum of the solid-fluid curve. The model also predicts a wider fluidfluid region, that is, an FF heterogeneity region of greater size, when the p-nitrobenzaldehyde concentration in the mixture increases. This can be seen in Figures $4 \mathrm{a}, \mathrm{d}-\mathrm{f}$.

It is worth noting that in this work we selected one of the simplest approaches to describe the phase behavior of the studied binary system, that is, we have used a classic equation of state (the Peng-Robinson EoS), associated with standard quadratic mixing rules to describe the behavior of the fluid phases, and we have regarded the solid phase as made of only pure p-nitrobenzaldehyde. From our modeling results, we conclude that the model basically succeeds in describing the observed behavior. However, a more complex approach could 
be used. For instance, the fluid state could be described through mixing rules that incorporate excess Gibbs free energy models; and the solid state could be represented through a model for solid solutions where both components would be present in the solid phase. However, the evaluation of all those possibilities is beyond the objectives of this work. In other words, the use of traditional mixing rules is justified in this work by their good observed performance in the description of the experimental data here obtained, performance which includes an observed consistent behavior well beyond the temperature and pressure conditions of such experimental data. Notice that despite their apparent simplicity, quadratic mixing rules are able to capture the main known behaviors of critical and three-phase lines of binary systems of widely varying level of asymmetry. ${ }^{48}$ Finally, using either complex mixing rules for the fluid state or the solid solution approach for the solid phase, or both, would increase the number of fitting parameters of the model.

\section{CONCLUSIONS}

In this work, solid-fluid equilibrium experimental data were obtained for the binary system $\mathrm{CO}_{2}(1)+$ p-nitrobenzaldehyde(2) through the static synthetic method. The ranges of temperature, pressure and p-nitrobenzaldehyde molar fractions studied were $281-353 \mathrm{~K}, 6.5-25.0 \mathrm{MPa}$, and $2.638 \times 10^{-3}$ to $5.903 \times 10^{-3}$, respectively. We observed that the pressure of solid-fluid transition increased with the increase in pnitrobenzaldehyde(2) concentration. In all the isopleths experimentally investigated, a minimum in the solid-fluid transition pressure was observed. The SF equilibrium of system $\mathrm{CO}_{2}(1)+$ p-nitrobenzaldehyde(2) was modeled through a simple approach. The model is able to capture the unusual phase behavior experimentally detected for $\mathrm{CO}_{2}(1)+\mathrm{p}$ nitrobenzaldehyde(2) mixtures, that is, the presence of local minima in the solid-fluid transition pressure (at a given overall composition). Calculations were performed in wide ranges of pressure and temperature to achieve a better understanding of the potential phase behavior of the $\mathrm{CO}_{2}(1)+$ p-nitrobenzaldehyde(2) system beyond the investigated experimental conditions, and to verify the absence of qualitatively inconsistent predictions.

\section{ASSOCIATED CONTENT}

\section{S Supporting Information}

The Supporting Information is available free of charge on the ACS Publications website at DOI: 10.1021/acs.jced.8b01067.

Binary solid-fluid equilibrium system of equations and description of type $\mathrm{F}$ phase behavior (PDF)

\section{AUTHOR INFORMATION}

\section{Corresponding Author}

*E-mail: franceschi.elton@gmail.com. Tel.: + 55-79-32182157. Fax: + 55-79-32182190.

\section{ORCID}

Marcelo S. Zabaloy: 0000-0002-8183-9523

Elton Franceschi: 0000-0002-2675-7250

\section{Funding}

We gratefully acknowledge the financial support provided by the following institutions from Brazil: CAPES (Coordination for the Improvement of Higher Education Personnel) Finance Code 001, CNPq (National Council for Scientific and Technological Development), FAPITEC/SE (Fundação de Apoio à Pesquisa e à Inovação Tecnológica do Estado de Sergipe), and FAPESP (Fundação de Amparo à Pesquisa do Estado de São Paulo), and from Argentina CONICET (PIP 11220150100918), ANPCyT (PICT-2017-1235), and UNS (PGI 24/M148).

\section{Notes}

The authors declare no competing financial interest.

\section{REFERENCES}

(1) Heravi, M. M.; Zadsirjan, V.; Dehghani, M.; Hosseintash, N. Current applications of organocatalysts in asymmetric aldol reactions: An update. Tetrahedron: Asymmetry 2017, 28, 587-707.

(2) Pansare, S.; Paul, E. The organocatalytic vinylogous aldol reaction: recent advances. Chem. - Eur. J. 2011, 17, 8770-8779.

(3) Bisai, V.; Bisai, A.; Singh, V. Enantioselective organocatalytic aldol reaction using small organic molecules. Tetrahedron 2012, 68, $4541-4580$

(4) Liu, L.; Liu, Z.-T.; Liu, Z.-W.; Xue, D. L-Proline catalyzed aldol reactions between acetone and aldehydes in supercritical fluids: An environmentally friendly reaction procedure. Sci. China: Chem. 2010, 53, 1586-1591.

(5) Li, L. Y.; Yang, D. C.; Guan, Z.; He, Y. H. Pepsin-catalyzed direct asymmetric aldol reactions for the synthesis of vicinal diol compounds. Tetrahedron 2015, 71, 1659-1667.

(6) Xie, Z. B.; Wang, N.; Jiang, G. F.; Yu, X. Q. Biocatalytic asymmetric aldol reaction in buffer solution. Tetrahedron Lett. 2013, 54, 945-948.

(7) Heravi, M. M.; Asadi, S. Recent applications of organocatalysts in asymmetric aldol reactions. Tetrahedron: Asymmetry 2012, 23, $1431-1465$

(8) Musko, N.; Grunwaldt, J. Heterogeneously catalyzed aldol reactions in supercritical carbon dioxide as innovative and nonflammable reaction medium. Top. Catal. 2011, 54, 1115-1123.

(9) Francio, G.; Wittmann, K.; Leitner, W. Highly efficient enantioselective catalysis in supercritical carbon dioxide using the perfluoroalkyl-substituted ligand (R,S)-3-H2F6-BINAPHOS. J. Organomet. Chem. 2001, 621, 130-142.

(10) Clarke, C. J.; Tu, W. C.; Levers, O.; Bröhl, A.; Hallett, J. P. Green and sustainable solvents in chemical processes. Chem. Rev. 2018, 118, 747-800.

(11) Bubalo, M. C.; Vidovic, S.; Redovnikovic, I. R.; Jokic, S. New perspective in extraction of plant biologically active compounds by green solvents. Food Bioprod. Process. 2018, 109, 52-73.

(12) Zhang, D.; Zhang, H.; Song, C.; Yang, W.; Deng, J. Chiral microspheres constructed by helical substituted polyacetylene: A new class organocatalyst toward asymmetric catalysis. Synth. Met. 2012, $162,1858-1863$.

(13) Cole-Hamilton, D. Asymmetric catalytic synthesis of organic compounds using metal complexes in supercritical fluids. Adv. Synth. Catal. 2006, 348, 1341-1351.

(14) Leitner, W.; Jessop, P. Supercritical Fluids, Vol. 4 of the Handbook of Green Chemistry; Anastas, P., Ed. (series); Wiley-VCH: Weinheim, 2010.

(15) Hagiwara, H.; Hamaya, J.; Hoshi, T.; Yokoyama, C. Self-aldol condensation of unmodified aldehyde in supercritical carbon dioxide catalyzed by amine grafted on silica. Tetrahedron Lett. 2005, 46, 393395.

(16) Grunwaldt, J.; Caravati, M.; Ramin, M.; Baiker, A. Probing active sites during palladium catalyzed alcohol oxidation in "supercritical" carbon dioxide. Catal. Lett. 2003, 90, 221-229.

(17) Debien, I.; Rigo, A.; Mazutti, M.; Oliveira, J.; Meireles, M. High-pressure phase equilibrium data for the l-lactic acid + (propane + ethanol) and the l-lactic acid + (carbon dioxide + ethanol) systems. J. Supercrit. Fluids 2013, 79, 27-31.

(18) Cassaro, R.; Oliveira, L.; Gariani, R.; Nascimento, C.; Bazito, R. Proline derivatives as organocatalysts for the aldol reaction in 
conventional and non-conventional reaction media. Green Process. Synth. 2013, 2, 43-50.

(19) Komoto, I.; Kobayashi, S. Lewis acid catalysis in a supercritical carbon dioxide $\left(\mathrm{scCO}_{2}\right)$-poly (ethylene glycol) derivatives (PEGs) system: remarkable effect of PEGS as additives on reactivity of $\mathrm{Ln}(\mathrm{OTf}) 3$ - catalyzed Mannich and aldol reactions in $\mathrm{scCO}_{2}$. Chem. Commun. 2001, 1842-1843.

(20) Mikami, K.; Matsukawa, S.; Kayaki, Y.; Ikariya, T. Asymmetric Mukaiyama aldol reaction of a ketene silyl acetal of thioester catalyzed by a binaphthol-titanium complex in supercritical fluoroform. Tetrahedron Lett. 2000, 41, 1931-1934.

(21) Kerton, F. Alternative Solvents for Green Chemistry; RSC Green Chemistry Book Series; RSC Publishing: Cambridge, 2009.

(22) Anastas, P.; Kirchhoff, M.; Williamson, T. Catalysis as a foundational pillar of green chemistry. Appl. Catal., A 2001, 221, 313.

(23) Fonseca, S.; Dohrn, R.; Peper, S. High-pressure fluid-phase equilibria: experimental methods and systems investigated (20052008). Fluid Phase Equilib. 2011, 300, 1-69.

(24) Dohrn, R.; Fonseca, J.; Peper, S. Experimental methods for phase equilibria at high pressures. Annu. Rev. Chem. Biomol. Eng. 2012, $3,343-367$.

(25) Pinto, L. F.; Rodriguez-Reartes, S. B.; Corazza, M. L.; Cabral, V. F.; Araújo, P. H. H.; Madureira, E. H.; Zabaloy, M. S.; Cardozo-Filho, L. Phase behavior of carbon dioxide + medroxyprogesterone acetate system at high pressures. Fluid Phase Equilib. 2013, 349, 1-11.

(26) Lucas, M. A.; Borges, G. R.; Rocha, I. C. C.; Santos, A. F.; Franceschi, E.; Dariva, C. Use of real crude oil fractions to describe the high pressure phase behavior of crude oil in carbon dioxide. J. Supercrit. Fluids 2016, 118, 140-147.

(27) Tenório Neto, E. T.; Kunita, M. H.; Rubira, A. F.; Leite, B. M.; Dariva, C.; Santos, A. F.; Fortuny, M.; Franceschi, E. Phase equilibria of the systems $\mathrm{CO}_{2}+$ styrene, $\mathrm{CO} 2+$ safrole and $\mathrm{CO} 2+$ styrene + safrole. J. Chem. Eng. Data 2013, 58, 1685-1691.

(28) Meneses, M. O.; Dariva, C.; Borges, G. R.; Da Rocha, S. R. P.; Zabaloy, M. S.; Franceschi, E. High-pressure phase behavior of poly(ethylene glycol) and 1,1,1,2-tetrafluorethane systems. J. Chem. Eng. Data 2017, 62, 1853-1858.

(29) Borges, G. R.; Junges, A.; Franceschi, E.; Corazza, F. C.; Corazza, M. L.; Oliveira, J. V.; Dariva, C. High-pressure vapor-liquid equilibrium data for systems involving carbon dioxide + organic solvent + b-carotene. J. Chem. Eng. Data 2007, 52, 1437-1441.

(30) Angus, S.; Armstrong, B.; Reuck, K. M. International Thermodynamics Tables of the Fluid State: Carbon Dioxide; Pergamon Press: Oxford, 1976.

(31) Suzuki, K.; Sue, H.; Itou, M.; Smith, R. L.; Inomata, H.; Arai, K.; Saito, S. Isothermal vapor-liquid equililbrium data for binary systems at high pressures: Carbon dioxide-methanol, carbon dioxideethanol, carbon dioxide-1-propanol, methane-ethanol, methane-1propanol, ethane-ethanol, and ethane-1-propanol systems. J. Chem. Eng. Data 1990, 35, 63-66.

(32) Day, C. Y.; Chang, C. J.; Chen, C. Y. Phase equililbrium of ethanol $+\mathrm{CO}_{2}$ and acetone $+\mathrm{CO}_{2}$ at elevated pressures. J. Chem. Eng. Data 1996, 41, 839-843.

(33) Secuianu, C.; Feroiu, F.; Gean, D. Phase behavior for carbon dioxide + ethanol system: Experimental measurements and modeling with a cubic equation of state. J. Supercrit. Fluids 2008, 47, 109-116. (34) Sima, S.; Feroiu, V.; Geana, D. New high pressure vapor-liquid equilibrium and density predictions for the carbon dioxide + ethanol system. J. Chem. Eng. Data 2011, 56, 5052-5059.

(35) Giufrida, W. M.; Rodriguez-Reartes, S. B.; Alonso, C. G.; Zabaloy, M. S.; Cabral, V. F.; Tavares, F. W.; Cardozo-Filho, L. Highpressure experimental data of $\mathrm{CO}_{2}+$ mitotane and $\mathrm{CO}_{2}+$ ethanol + mitotane mixtures. J. Chem. Eng. Data 2011, 56, 4333-4341.

(36) Rodriguez-Reartes, S. B.; Cismondi, M.; Franceschi, E.; Corazza, M. L.; Oliveira, J. V.; Zabaloy, M. S. High-pressure phase equilibria of systems carbon dioxide $+n$-eicosane and propane $+n-$ eicosane. J. Supercrit. Fluids 2009, 50, 193-202.
(37) Peng, D. Y.; Robinson, D. B. A new two-constant equation of state. Ind. Eng. Chem. Fundam. 1976, 15, 59-64.

(38) Michelsen, M. L.; Mollerup, J. M. Thermodynamic Models: Fundamentals and Computational Aspects; Tie-Line Publications: Holte, Denmark, 2004.

(39) Rodriguez-Reartes, S. B.; Cismondi, M.; Zabaloy, M. S. Computation of solid-fluid-fluid equilibria for binary asymmetric mixtures in wide ranges of conditions. J. Supercrit. Fluids 2011, 57, 924.

(40) DIPPR-801. Evaluated Process Design Data, Public Release. American Institute of Chemical Engineers, Design Institute for Physical Property Data, BYU-DIPPR. Thermophysical Properties Laboratory: Provo, UT, 2003.

(41) Hukkerikar, A. S.; Sarup, B.; Kate, A. T.; Abildskov, J.; Sin, G.; Gani, R. Group-contribution+ $(\mathrm{GC}+)$ based estimation of properties of pure components: Improved property estimation and uncertainty analysis. Fluid Phase Equilib. 2012, 321, 25-43.

(42) Goodman, B. T.; Wilding, W. V.; Oscarson, J. L.; Rowley, R. L. A note on the relationship between organic solid density and liquid density at the triple point. J. Chem. Eng. Data 2004, 49, 1512-1514.

(43) Yaws, C. L. The Yaws Handbook of Physical Properties for Hydrocarbons and Chemicals: Physical Properties for More Than 54,000 Organic and Inorganic Chemical Compounds, Coverage for C1 to C100 Organics and Ac to Zr Inorganics; Elsevier: Amsterdam, 2015.

(44) Dandge, D. K.; Heller, J. P.; Wilson, K. V. Structure solubility correlations: organic compounds and dense carbon dioxide binary systems. Ind. Eng. Chem. Prod. Res. Dev. 1985, 24, 162-166.

(45) Yamamoto, S.; Kazunari, O.; Takashi, K. Phase behavior of binary mixtures of indole or quinoxaline with $\mathrm{CO}_{2}, \mathrm{C}_{2} \mathrm{H}_{4}, \mathrm{C}_{2} \mathrm{H}_{6}$, and $\mathrm{CHF}_{3}$ in the critical region. J. Supercrit. Fluids 1989, 2, 63-72.

(46) Prausnitz, J. M.; Lichtenthaler, R. N.; Gomes de Azevedo, E. Molecular Thermodynamics of Fluid-phase Equilibria, 3rd ed.; PrenticeHall PTR: NJ, 1999.

(47) Lusi, M. A rough guide to molecular solid solutions: design, synthesis and characterization of mixed crystals. CrystEngComm 2018, 20, 7042-7052.

(48) Scott, R. L.; Van Konynenburg, P. H. Static properties of solutions: Van der Waals and related models for hydrocarbon mixtures. Discuss. Faraday Soc. 1970, 49, 87-97. 